\title{
Triple Conjoined Nerve Root-An Unreported Anatomical Variant and Its Surgical Implications
}

\author{
Kelly Gassie ${ }^{1}$ Krishnan Ravindran ${ }^{2}$ Gazanfar Rahmathulla²
}

\author{
H. Gordon Deen J $r^{1}$
}

\begin{abstract}
Address for correspondence Gazanfar Rahmathulla, MD (DNB Neurosurgery), FACS, IFAANS, MBA, Department of Neurosurgery, University of Florida College of Medicine, 580W 8th Street, 8th Floor, Clinical Tower 1, Jacksonville, FL 32209, United States (e-mail: Gazanfar.rahmathulla@jax.ufl.edu).
\end{abstract}

\begin{abstract}
Keywords

- conjoined nerve root

- radiculopathy

- anatomical variant

- implications

Conjoined nerve roots are an infrequent and uncommon finding, rarely noted preoperatively. The conjoined root anomaly has potential for significant neurological injury during surgery. Preoperative recognition may avert disastrous nerve root injury but requires a high degree of clinical suspicion. We present the case of a 44-year-old patient with left L5/S1 radiculopathy caused by a herniated disc. During surgery we identified a triple conjoined nerve root anatomy. This anatomical variant, to our knowledge, has not been reported in literature. We describe the anatomical findings and surgical implications.
\end{abstract}

\section{Introduction}

When two adjacent nerve roots share a common dural envelope at some point along their course, they are referred to as a conjoined nerve root. ${ }^{1}$ These are more commonly found unilaterally, but reports of bilateral conjoined roots have also been described., ${ }^{2,3}$ There are several physical exam findings and imaging signs reported in the literature, but none of these findings are diagnostic of this anomalous anatomy. ${ }^{4}$ The embryological mechanism for the development of conjoined roots is hypothesized to be secondary to a root migration defect ${ }^{5,6}$ with the exact details yet to be clarified. Cannon et $\mathrm{al}^{7}$ classified conjoined roots into three types based on their anatomical characteristics in relation to the dural sheath, pedicle, and foraminal course. Modifications to this classification have been made by Neidre and Macnab, ${ }^{8}$ with Postacchini et $\mathrm{al}^{9}$ making further changes to the lumbosacral anomalies based on operative findings. More recently Kadish and Simmons ${ }^{10}$ made further changes based on the anatomy and radiological findings and outlined four types of conjoined roots in their classification.

We describe a case of a 44-year-old female patient presenting with characteristics of an L5 and S1 radiculopathy

DOI https://doi.org/ $10.1055 / \mathrm{s}-0040-1713056$ ISSN 2277-954X. with an extruded L5/S1 disc. We report a triple conjoined nerve root that was identified during surgery and describe the anatomical findings and implications of such variations at surgery.

\section{Case Report}

A 44-year-old female patient presented with a history of long standing left lower extremity radiculopathy without any neurological deficits. She had attempted conservative medical management, including chiropractic manipulation, physical therapy, and pain management for over 6 months without any symptomatic relief. - Fig. 1 describes a magnetic resonance imaging (MRI) of lumbar spine revealing a herniated L5/S1 disc.

She underwent a left-sided hemilaminectomy; during the procedure it was difficult to manipulate the traversing nerve root. On further dissection there were two other roots running adjacent to it. To delineate the anatomy more clearly an extension of the hemilaminectomy was performed laterally, and a resection of the medial facet joint had to be done. The exiting nerve root was visualized running below the pedicle of L5, arising from a common dural origin along with two other traversing 
nerve roots, making it a triple conjoined root anomaly (-Fig. 2). The dural sheath between the roots was dissected to identify the disc space and an adjacent disc bulge; however, adequate safe access could not be obtained with this maneuver (-Fig. 3). On further review of her preoperative images during the surgery, we were able to

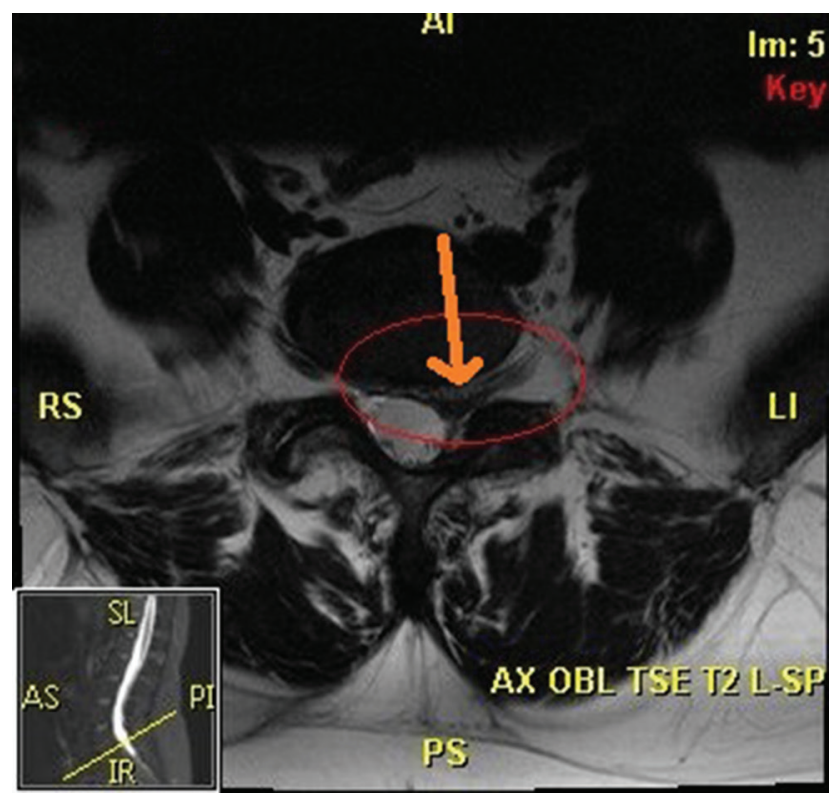

Fig. 1 Axial MR-T2W image revealing the large herniated disc fragment in the foraminal L5/S1 space (within the red oval, orange arrow points to disc). MR-T2W, magnetic resonance-T2 weighted.

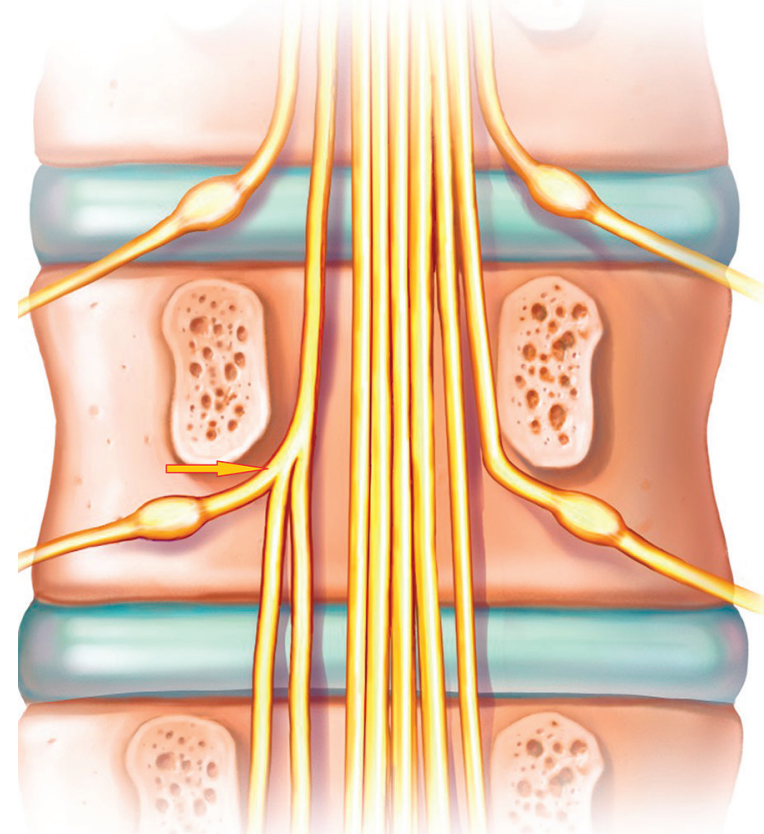

A

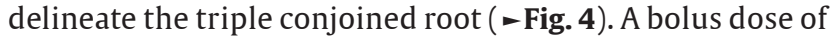
$10 \mathrm{mg}$ of intravenous dexamethasone was given and the roots were gently retracted medially to get access to the disc space, from a far lateral angle made possible because of the medial facetectomy, resecting approximately twothirds of the facet to provide a more lateral route and reduce nerve root retraction, followed by a routine microsurgical discectomy being performed (-Fig. 5). Her postoperative recovery was uneventful with complete pain relief maintained at 12 weeks of follow-up.

\section{Discussion}

The most common level at which conjoined roots has been identified are $\mathrm{L} 5 / \mathrm{S} 1$. White et $\mathrm{al}^{11}$ reported an incidence of $1.3 \%$, with similar rates from other reports in literature. ${ }^{12}$ Imaging studies like computerized tomography scans have reported incidences of $2 \%$. $^{13,14}$

MRI scans are presently the most commonly used imaging modality to detect conjoined roots. ${ }^{15-17}$ Various signs on routine sagittal and axial MRI scans have been described such as Kang et al sagittal shoulder sign and the axial sagittal passage sign. ${ }^{18}$ Song et al described the corner sign; others include the fat crescent sign and the parallel sign, all of which have been well described in other publications. ${ }^{19}$ At times conjoined roots may also be difficult to be differentiated from herniated lumbar discs at these levels.

In this report we describe a triple root variant of a conjoined root, previously unreported in peer-reviewed

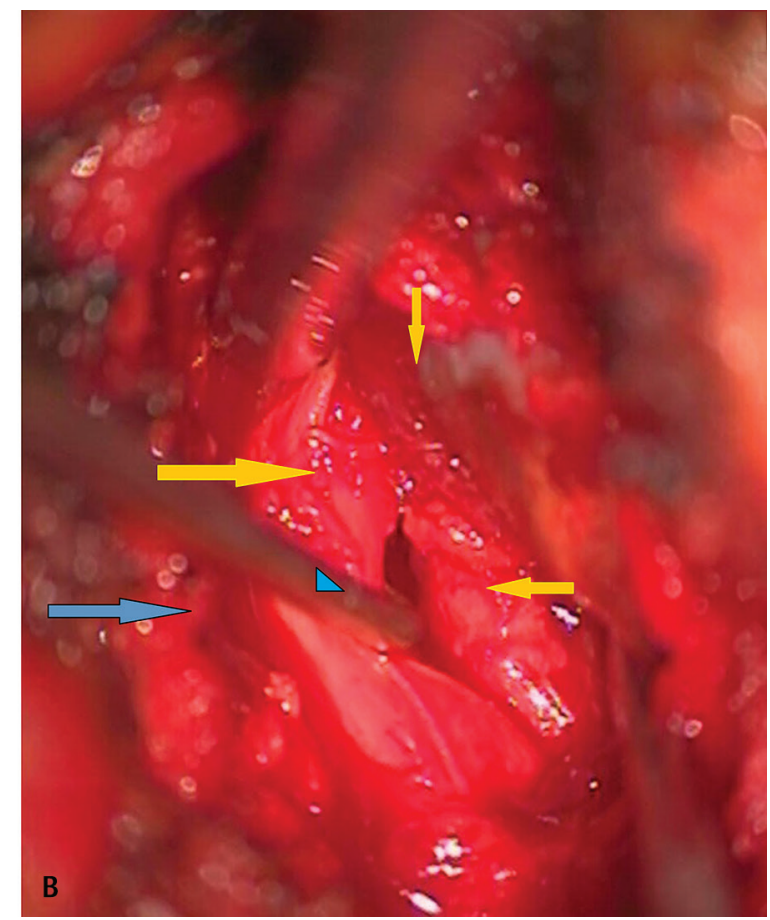

Fig. 2 (A) Representative illustration of the triple root anomaly (yellow arrow pointing to the origin) clearly revealing what the authors visualized, as the microscopic images only reveal the traversing rootlets and not the L5 exiting root. (B) Intraoperative image of the L5/S1 foraminal level (gray arrow lateral and directed to facet joint) widely dissected out (blue triangle arrowhead over the nerve root retractor) to delineate the anatomy. The L5 nerve root exits below the L5 pedicle at right angles to the parallel S1 roots running inferiorly (yellow arrows pointing medial and lateral revealing the nerve roots), with all three originating in a common dural sheath above (yellow arrow pointing inferiorly). 


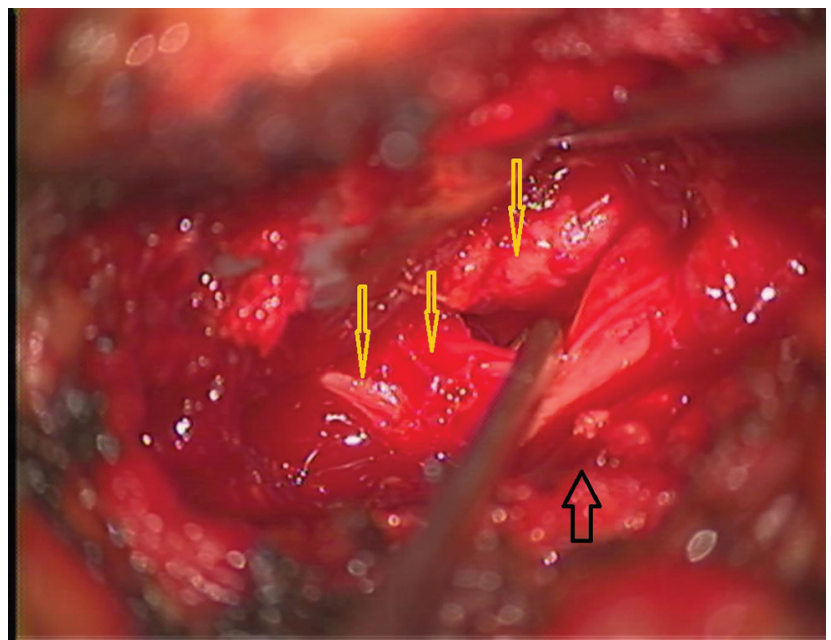

Fig. 3 The S1 roots have been separated out with a blunt nerve hook revealing the difficulty in retracting these roots (yellow arrows), making it difficult to clearly delineate and excise the underlying disc. In this case we extended our lateral bony resection to the medial twothirds of the facet joint (black arrow) enabling a more lateral approach and exposure of the disc space.

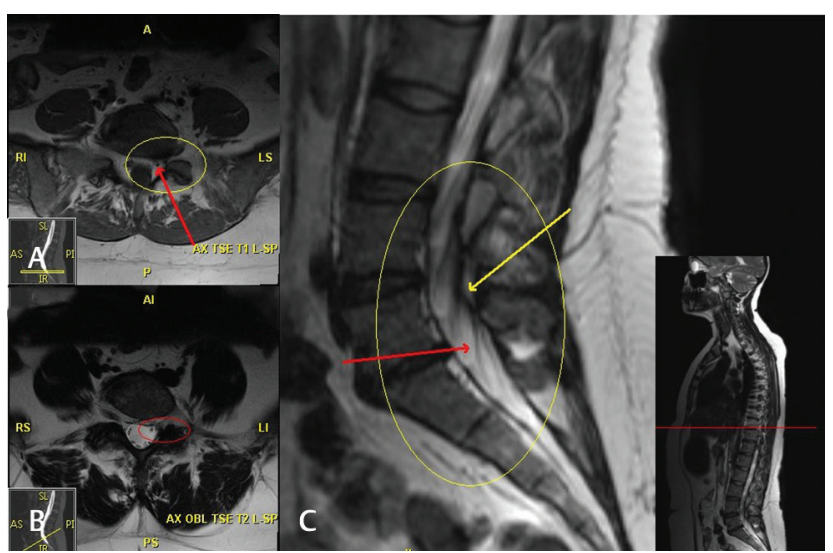

Fig. 4 Axial images T1 weighted at the disk level (A, B) and sagittal MR-T2W image $(C)$ revealing the anomalous anatomy (within the yellow oval) and the common origin of the triple conjoined root (yellow arrow) as well as an anomalous distal bifurcation (red arrow). MR-T2W, magnetic resonance-T2 weighted.

indexed spinal literature. We have found a Senegalese study with an unclassified variant which could possibly be similar to our case and if so, ours would be the second reported case of a triple conjoined root variant. ${ }^{20}$ Trimba et $\mathrm{al}^{4}$ have discussed the various types and classifications of conjoined roots over the years in their recent review article. The conjoined roots are usually fixed in position and retraction may induce injury and inflammation giving rise to temporary or permanent neurological deficits. Due to the adhesions of the dural sheath at various points along the course of these roots, attempting to separate or dissect them out with blunt or sharp dissection could give rise to dural tears and cerebrospinal fluid leaks. If the surgeon does not consider the possibility of a conjoined root, inadvertent injury may occur by incorrectly assuming root for disc and sharply incising

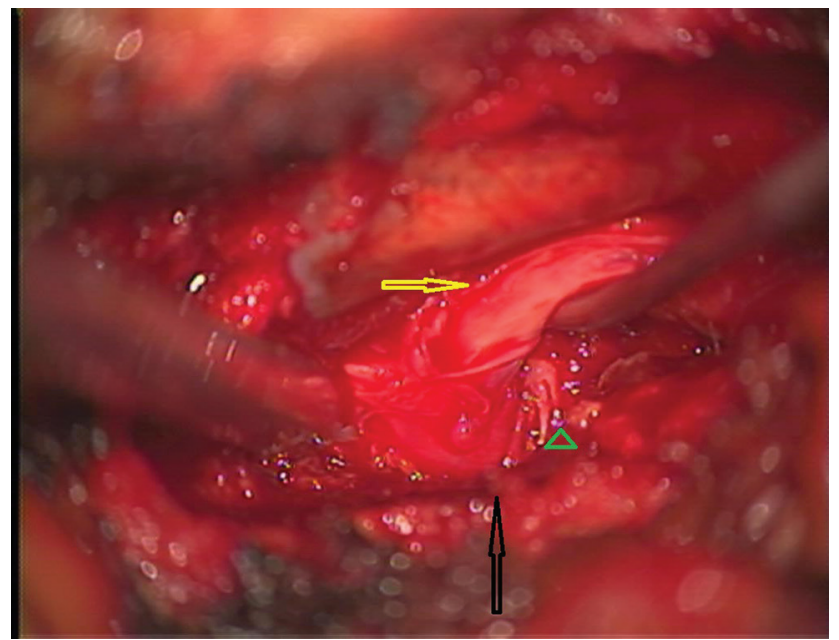

Fig. 5 The medial facetectomy (black arrow) enabled a more lateral visualization and approach, allowing gentle traction (yellow arrow), delineating the disc fragment (brown arrowhead) and a microsurgical excision of the disc fragment.

this may give rise to permanent deficits..$^{1,8,18,19}$ Various publications have reported performing procedures ranging from a hemilaminectomy, lateral positioning, and approach to the disc and, extended bone resection laterally to include the facet and even the pedicle above and below to get good exposure and visualization. $5,7,11,21,22$ In rare cases where the nerve root arises at right angles, adequate exposure necessitating pedicle resection may be essential to carry out the procedure without damaging these nerve roots. ${ }^{7}$ We did resect the hemilamina as extensively required to avoid any root retraction. Additionally, we removed the overhanging facets which would give us more space to approach the disc space laterally. We did not feel the necessity of adding instrumentation to stabilize her at surgery. The disc fragment was excised, and the nerve roots decompressed. No pedicle resection was performed in this procedure, although it has been advocated for cases with anomalous lumbar nerve root anatomy. The patient will be followed for any delayed onset of instability both with clinical and radiological examinations and if required will intervene with instrumented arthrodesis at that time.

\section{Conclusion}

The preoperative recognition and diagnosis of a conjoined root anomaly is extremely unlikely. Ours is possibly the first such report of a triple conjoined root anomaly, but more importantly, alternate surgical strategies are safe and effective, in case the surgeon identifies anomalous nerve anatomy on the operating table. A triple conjoined root provides little spaces for retraction and endoscopic and minimally invasive surgery could cause neurological injury. A high degree of astuteness in the identification and reporting of these variants along with adapting surgical techniques intraoperatively is necessary to treat these variations safely and effectively. 


\section{Conflict of Interest}

None declared.

\section{References}

1 Scuderi GJ, Vaccaro AR, Brusovanik GV, Kwon BK, Berta SC. Conjoined lumbar nerve roots: a frequently underappreciated congenital abnormality. J Spinal Disord Tech 2004;17(2):86-93

2 Gambardella A, Simari Benigno R, Mazza M, Smaltino F. Anomalies of the lumbosacral nerve roots [in Italian]. Radiol Med (Torino) 1985;71(1-2):36-39

3 Helms CA, Dorwart RH, Gray M. The CT appearance of conjoined nerve roots and differentiation from a herniated nucleus pulposus. Radiology 1982;144(4):803-807

4 Trimba R, Spivak JM, Bendo JA. Conjoined nerve roots of the lumbar spine. Spine J 2012;12(6):515-524

5 Artico M, Carloia S, Piacentini M, et al. Conjoined lumbosacral nerve roots: observations on three cases and review of the literature. Neurocirugia (Astur) 2006;17(1):54-59

6 Bouchard JM, Copty M, Langelier R. Preoperative diagnosis of conjoined roots anomaly with herniated lumbar disks. Surg Neurol 1978;10(4):229-231

7 Cannon BW, Hunter SE, Picaza JA. Nerve-root anomalies in lumbar-disc surgery. J Neurosurg 1962;19:208-214

8 Neidre A, MacNab I. Anomalies of the lumbosacral nerve roots. Review of 16 cases and classification. Spine 1983;8(3):294-299

9 Postacchini F, Urso S, Ferro L. Lumbosacral nerve-root anomalies. J Bone Joint Surg Am 1982;64(5):721-729

10 Kadish LJ, Simmons EH. Anomalies of the lumbosacral nerve roots. An anatomical investigation and myelographic study. J Bone Joint Surg Br 1984;66(3):411-416

11 White JG III, Strait TA, Binkley JR, Hunter SE. Surgical treatment of 63 cases of conjoined nerve roots. J Neurosurg 1982;56(1): $114-117$
12 Coughlin JR, Miller JD. Metrizamide myelography in conjoined lumbosacral nerve roots. J Can Assoc Radiol 1983;34(1):23-25

13 Torricelli P, Spina V, Martinelli C. CT diagnosis of lumbosacral conjoined nerve roots. Findings in 19 cases. Neuroradiology 1987;29(4):374-379

14 Peyster RG, Teplick JG, Haskin ME. Computed tomography of lumbosacral conjoined nerve root anomalies. Potential cause of false-positive reading for herniated nucleus pulposus. Spine 1985;10(4):331-337

15 Böttcher J, Petrovitch A, Sörös P, Malich A, Hussein S, Kaiser WA. Conjoined lumbosacral nerve roots: current aspects of diagnosis. Eur Spine J 2004;13(2):147-151

16 Savas R, Calli C, Yünten N, Alper H. Hypoplastic lumbar pedicle in association with conjoined nerve root MRI demonstration. Comput Med Imaging Graph 1998 22(1):77-79

17 Haijiao W, Koti M, Smith FW, Wardlaw D. Diagnosis of lumbosacral nerve root anomalies by magnetic resonance imaging. J Spinal Disord 2001;14(2):143-149

18 Kang $\mathrm{CH}$, Shin MJ, Kim SM, et al. Conjoined lumbosacral nerve roots compromised by disk herniation: sagittal shoulder sign for the preoperative diagnosis. Skeletal Radiol 2008;37(3):225-231

19 Song SJ, Lee JW, Choi JY, et al. Imaging features suggestive of a conjoined nerve root on routine axial MRI. Skeletal Radiol 2008;37(2):133-138

20 Kessely YC, Sakho MG, Fondo A, Daisy AD, Thiam A, Sakho Y. Conjoined nerve root: about a case [in French]. Pan Afr Med J 2015;20:166

21 Pamir MN, Ozek MM, Ozer AF, Keles GE, Erzen C. Surgical considerations in patients with lumbar spinal root anomalies. Paraplegia 1992;30(5):370-375

22 Epstein JA, Carras R, Ferrar J, Hyman RA, Khan A. Conjoined lumbosacral nerve roots. Management of herniated discs and lateral recess stenosis in patients with this anomaly. J Neurosurg 1981;55(4):585-589 